\title{
Scaling Properties of the Internet Graph
}

\author{
Aditya Akella ${ }^{1} \quad$ Shuchi Chawla ${ }^{2} \quad$ Arvind Kannan $^{3}$ \\ Srinivasan Seshan ${ }^{4}$ \\ May 2003 \\ CMU-CS-03-143 3
}

School of Computer Science

Carnegie Mellon University

Pittsburgh, PA 15213

\footnotetext{
${ }^{1}$ Computer Science Department, Carnegie Mellon University (aditya@cs.cmu.edu).

${ }^{2}$ Computer Science Department, Carnegie Mellon University (shuchi@cs.cmu.edu).

${ }^{3}$ Computer Science Department, Carnegie Mellon University (arvind@cmu.edu).

${ }^{4}$ Computer Science Department, Carnegie Mellon University (srini@cmu.edu).
} 
Keywords: Power-law Distribution, Congestion, Shortest Path Routing, Policy Routing 


\begin{abstract}
As the Internet grows in size, it becomes crucial to understand how the speeds of links in the network must improve in order to sustain the pressure of new end-nodes being added each day. Although the speeds of links in the core and at the edges roughly improve according to Moore's law, this improvement alone might not be enough. Indeed, the structure of the Internet graph and routing in the network might necessitate much faster improvements in the speeds of key links in the network.

In this paper, using a combination of analysis and extensive simulations, we show that the worst congestion in the Internet in fact scales poorly with the network size $\left(n^{1+\Omega(1)}\right.$, where $n$ is the number of nodes), when shortest-path routing is used. We also show, somewhat surprisingly, that policy-based routing does not exacerbate the maximum congestion when compared to shortest-path routing.

Our results show that it is crucial to identify ways to alleviate this congestion to avoid some links from being perpetually congested. To this end, we show that the congestion scaling properties of the Internet graph can be improved dramatically by introducing moderate amounts of redundancy in the graph in terms of parallel edges between pairs of adjacent nodes.
\end{abstract}





\section{Introduction}

The Internet grows in size every day. As time progresses, more end-hosts are added to the edge of the network. Correspondingly, to accommodate these new end-hosts, ISPs add more routers and links. History has shown that the addition of these links maintains the power law properties of the Internet topology [8]. The addition of new end-hosts places a greater load on the network as a whole. Fortunately, the improvement of network technology, operates over the same time period. We expect the network links at the edge and core of the network to improve by a similar performance factor as the growth of traffic over time, since they both typically follow similar Moore's Law-like technology trends.

Unfortunately, due to the topology of the network and behavior of Internet routing, the increase in load may be different on different links. As a result, it may be necessary for the speed of some hot-spot links in the network to improve much more quickly than others. If this is true, then these parts of the network are likely to eventually become bottlenecks and the network has poor scaling properties. In such a situation, we would either need to adjust the routing behavior, remove the power law nature of the topology or accept that end-to-end network performance of the network will not improve as rapidly as individual links. If, on the other hand, the worst congestion scales well with the network size then we can expect the network to continue to operate as it does now.

In this paper, we use a combination of analysis and simulation to address this issue of how the maximum congestion in the Internet scales with the network size. In our analysis, we employ simple combinatorial/probabilistic arguments to give bounds on the maximum congestion in a model of network evolution based on Preferential Connectivity [6] and a simple model of traffic in which a unit amount of flow between every pair of nodes is routed along the shortest path between them. We complement these analytical results with a large set of detailed simulations for computing the congestion on the links in the network, based both on real and on synthetically generated $A S$-level topologies. Through our simulations, we also investigate the impact of several key factors on the worst congestion in the network. These include:

1. The routing algorithm employed by the nodes in the network: BGP policy-driven routing (which we refer to as policy routing) vs. shortest-path routing,

2. Sophisticated models of communication between nodes, modeling realistic traffic demands and factoring in the higher popularity of a few nodes in the network over the others, and,

3. Alternate degree distributions, e.g., exponential distribution and power law trees evolving from Preferential Connectivity.

The key contribution of our paper is to show that the maximum congestion in the Internet scales poorly with the growing size of the Internet graph. Specifically, the maximum congestion for shortest path routing is at least as bad as $n^{1+\Omega(1)}$, with the exponent depending on the exponent of the power law degree distribution of the graph ${ }^{1}$. Our simulations show that policy routing in the AS graph results in roughly the same maximum congestion as shortest path routing, but certainly not worse. When more realistic, non-uniform traffic models are considered, the congestion scaling properties of power law graphs worsen substantially. We also show that in terms of the maximum congestion, power law trees are considerably worse than power law graphs. In contrast, graphs with exponential degree distribution have very good congestion properties.

Another key contribution of our paper is the discussion of simple guidelines that result in a dramatic improvement in the congestion scaling properties of the Internet graph. We show that when parallel links are added between adjacent nodes in the network according to simple functions of their degrees (e.g., the minimum of the two degrees), the maximum congestion in the resulting graph scales linearly.

The rest of the paper is structured as follows. We discuss related work in Section 2. In Section 3, we formalize our analytical approach and discuss our simulation set-up. The analysis is presented in Section 4 . Section 5 presents the results from our simulations. In Section 6, we discuss the implications of our results on network design. Finally, in Section 7, we conclude the paper.

\footnotetext{
${ }^{1}$ There is some disagreement about whether a power law correctly models the degree distribution of the Internet graph. However, it is widely agreed that the distribution is heavy-tailed. While our main results (specifically, simulation results) focus on power law distributions, we believe that they hold equally well for other such heavy-tailed distributions (e.g. Weibull).
} 


\section{Related Work}

In the past, there have been several research efforts aimed at studying the properties of large-scale, Internetlike graphs. Of these, one class of studies has proposed various models of graph evolution that result in a power law degree distribution. Notable examples include the power law random graph model of Aiello et. al. [3], the bicriteria optimization model of Fabrikant et. al. [7] and the Preferential Connectivity model of Barabasi and Albert [6,4]. Another class of studies in this category $[8,16,18]$ is aimed at analyzing the properties of power law graphs. However, most of these are based on inferences drawn from measurements of real data. Other efforts $[13,19,18]$ have used these inferences to construct realistic generators for Internet-like graphs. Our theoretical analysis is based on the Preferential Connectivity model of Barabasi and Albert [6]. Our simulations use topologies generated synthetically using Inet-3.0 [19].

The problem of characterizing congestion in graphs, and specifically designing routing schemes that minimize congestion, has been studied widely in approximation and online algorithms. The worst congestion in a graph is inversely related to the maximum concurrent flow that can be achieved in the graph while obeying unit edge capacities. The latter is, in turn, related to a quantity called the cut ratio of the graph. Aumann et. al. [5] characterize the relationship between maximum concurrent flow and cut ratio ${ }^{2}$ and Okamura et. al. [15] give bounds on the cut ratio for special graphs. Algorithmic approaches to the problem (see $[11,12]$ for a survey) use a multi-commodity flow relaxation of the problem to find a fractional routing with good congestion properties. Although fairly good approximation factors have been achieved for the problem, most of these are not distributed, involve a lot of book-keeping, or involve solving large linear programs, which makes them impractical from the point of view of routing on the Internet. Therefore, we choose the approach of analyzing the congestion achieved from using widely implemented routing schemes such as shortest path or BGP-policy based routing.

Perhaps the work that bears closest resemblance to ours is that of Mihail et. al. [10]. Using arguments from max-flow min-cut theory, their paper shows that graphs obeying power law degree distribution have good expansion properties, in that they allow routing with $O\left(n \log ^{2} n\right)$ congestion, which is close to the optimal value of $O(n \log n)$ achieved by regular expanders. In addition, based on simulations run over Inet-generated topologies, the paper concludes that the congestion in power law graphs scales almost as $O\left(n \log ^{2} n\right)$, even when shortest path routing is used. The paper also shows that policy routing results in worse congestion.

Our work is different from [10] in several key aspects, a few of which we identify below. First, the theoretical analysis in [10] does not restrict the routing to shortest path and, in fact, assumes an optimal routing algorithm that minimizes congestion. We show that, in fact when shortest path routing is employed, power law graphs exhibit poor scaling properties in terms of congestion. The maximum congestion scales as $n^{1+\Omega(1)}$. We confirm this via detailed simulation experiments. In addition, our simulations also show that policy routing does not worsen the maximum congestion in the network contrary to the conclusion in [10]. The evaluations of policy routing and shortest path routing in [10] only consider graphs with a small number of nodes, approximately 10,000 nodes for policy routing graphs (due to the dependence on real AS graphs) and only 23,000 for the shortest path routing graphs. Our simulations, on the other hand, consider graphs of up to 50000 nodes. Finally, we also consider the impact of different traffic workloads and deployments of parallel links on the scaling properties of the network.

\section{Methodology}

We use combinatorial/probabilistic arguments over a simple model of the network combined with extensive simulations to understand the congestion properties of the Internet graph. In what follows, we first give a precise formulation of the problem, laying out the key questions we seek to address via analysis. We also describe the set-up for the simulations we use to corroborate and extend our analytical arguments.

\footnotetext{
${ }^{2}$ The maximum concurrent flow that can be achieved in a graph is always within a factor of $O(\log n)$ of the cut ratio, where $n$ is the number of nodes.
} 


\subsection{Problem Statement}

Let $G=(V, E)$ be an unweighted graph, representing the Internet AS-AS graph, with $|V|=n$. Let $d_{v}$ denote the total degree of a vertex $v$ in $G$. We are given three key aspects pertaining to the graph $G$ : the degree distribution of the graph, the routing algorithm used by the nodes in the graph to communicate with each other and the traffic demand matrix determining the amount of traffic between pairs of nodes in the graphs. We give precise definitions of these three aspects, in turn, below.

In our paper we will mostly be concerned with graphs having a power law degree distribution, defined below.

Definition 1 We say that an unweighted graph $G$ has a power law degree distribution with exponent $\alpha$, if for all integers $d$, the number of nodes $v$ with $d_{v} \geq d$ is proportional to $d^{-\alpha}$.

Similarly, graphs with exponential degree distribution are those in which the number of nodes $v$ with $d_{v} \geq d$ is proportional to $e^{-\beta d}$, for all integers $d$. Henceforth, we will refer to such graphs as power law graphs and exponential graphs respectively.

Let $\mathcal{S}$ denote a routing scheme on the graph with $\mathcal{S}_{u, v}$ representing the path for routing traffic between nodes $u$ and $v$. We consider two different routing schemes in this paper:

1. Shortest Path Routing: In this scheme, the route between nodes $u$ and $v$ is given by the shortest path between the two nodes in the graph $G$. When there are multiple shortest paths, we consider the maximum degree of nodes along the paths and pick the one with the highest maximum degree. This tie-breaking rule is reflective of the typical policies employed in the Internet-higher degree nodes are typically much larger and much more well-provisioned providers than lower degree nodes and are in general used as the primary connection by stub networks. Additionally, to be complete in the situations that we assess, we consider breaking ties by favoring lower degree nodes and also by choosing random shortest paths.

We report results for all three of the above tie-breaking rules and show that the results are fairly consistent across the three schemes.

2. Policy Routing: In this scheme, traffic between nodes $u$ and $v$ is routed according to BGP-policy. We classify edges as peering edges or customer-provider edges (that is, one of the ASes is a provider of the other). Typically, ASes in the Internet only provide transit for traffic destined to their customers, if any. This implies that no AS will carry traffic from its peer to another of its peers or to its provider. Similarly, no AS will carry traffic from one of its providers to one of its peers or to its provider. These rules together give rise to "valley-free" routing, in which each path contains a sequence of customer to provider edges, followed by at most one peering edge, followed by provider to customer edges. For a detailed description of the mechanism, the reader is referred to [17].

A traffic vector $\tau$ is a vector containing $\left(\begin{array}{l}n \\ 2\end{array}\right)$ non-negative terms, with the term corresponding to $(u, v)$ signifying the amount of traffic between the nodes $u$ and $v$. The congestion on an edge $e$ due to traffic vector $\tau$ and routing scheme $\mathcal{S}$ is given by the sum of the total amount of traffic that uses the edge $e$ : $\mathcal{C}_{\tau, \mathcal{S}}(e)=\sum_{(u, v): e \in \mathcal{S}_{u, v}} \tau(u, v)$.

We define the edge congestion due to traffic vector $\tau$ and routing scheme $\mathcal{S}$ to be the maximum congestion on any edge in the graph:

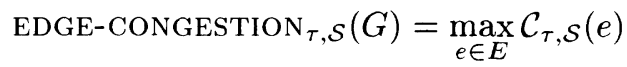

In this paper, we are interested in quantifying the congestion in a graph with power law degree distribution, for shortest path and policy routing schemes, due to various different traffic vectors. Specifically, we consider the following three traffic vectors:

1. Any-2-any: This corresponds to the all 1 s traffic vector, with a unit traffic between every pair of nodes.

2. Leaf-2-leaf: In order to define this model, we classify nodes in the graph as stubs and carriers. Stubs are nodes that do not have any customers. In other words, consider directing all customer-provider edges in the graph from the customer to the provider. Peering edges are considered to be bidirected 
edges. Then, vertices with no incoming edges (corresponding to ASes with no customers) are called stubs or leaves in the graph. In this model, there is a unit of traffic between every pair of stubs in the graph.

3. Clout: This model is motivated by the fact that "well-placed" sources, that is, sources that have a high degree and are connected to high degree neighbors, are likely to send larger amounts of traffic than other sources. Accordingly, in this case, $\tau_{(u, v)}=f\left(d_{u}, c_{u}\right)$, where $u$ and $v$ are stubs, $c_{u}$ is the average degree of the neighbors of $u$ and $f$ is an increasing function. As in the previous case, there is no traffic between nodes that are not stubs. In this paper, we only use the function $\tau_{(u, v)}=f\left(d_{u}, c_{u}\right)=d_{u} c_{u}$ for stubs $u, v$.

\subsection{Simulation Setup}

Our simulations serve two purposes: (1) to corroborate our theoretical results, and, (2) to characterize the congestion in more realistic network models than those considered in our analysis.

Our simulations are run on two different sets of graphs. The first set of graphs contains maps of the Internet AS topology collected at 6 month intervals between Nov. 1997 and April 2002, available at [2]. The number of nodes in any graph in this set is at most 13000, the maximum corresponding to the April 2002 set. The second set of graphs contains synthetic power law graphs generated by Inet-3.0 [19]. In this set, we generate graphs of sizes varying from $n=4000$ to 50000 nodes. In all our simulations, for any metric of interest, for each $n$, we generate 5 slightly different graphs of $n$ nodes $^{3}$ and report the average of the metric on the 5 graphs.

As pointed out in Section 3.1, in order to implement the leaf-2-leaf and clout models of communication, we need to identify stubs in the network (note that these might have a degree greater than 1). Additionally, in order to implement policy routing, we need to classify edges as peering or non-peering edges. In order to do so, for the real AS graphs, we employ the relationship inference algorithms of Gao [9] to label the edges of the graphs as peering or customer-provider edges. These algorithms use global BGP tables [1] to infer relationships between nodes. Then, we use these relationships to identify stubs (as nodes that are not providers of any other node). Henceforth, we shall refer to the real AS graphs as accurately labeled real graphs (ALRs). Labeling edges and identifying stubs in the synthetic graphs of Inet is more tricky since we do not have the corresponding BGP information. We will refer to synthetic graphs, labeled using the algorithms described below, as heuristically labeled synthetic graphs (HLSs). We use different algorithms for classifying nodes (this is key to implementing leaf-to-leaf communication) and edges (this is key to implementing policy routing in synthetic graphs). We discuss each in turn below.

\section{Stub Identification}

Here is how we identify stubs in synthetic graphs: For any edge $e=\left(v_{1}, v_{2}\right)$, we assign $v_{1}$ to be the provider of $v_{2}$ whenever degree $\left(v_{1}\right) \geq \operatorname{degree}\left(v_{2}\right)$. Notice that we do not explicitly identify peering edges (although edges between nodes of identical degree will be bidirectional). We then identify stubs in graphs labeled as above.

We test the accuracy of this stub-identification algorithm on real AS graphs by comparing the labels produced by our algorithm to the true labels of ALRs, and compute the fraction of false positives and false negatives $^{4}$ in these. The results (see Figure 1(a)) show that our simple algorithm has very low error rate. Notice that the inference algorithms of Gao [9] have some error intrinsically and hence some of the labels on the ALRs might actually be inaccurate.

\section{Edge Classification}

Although for the purpose of classifying nodes, we simply consider all edges in the graph to be customerprovider edges, this simple scheme is not useful for the purposes of edge classification - it results in a

\footnotetext{
${ }^{3} \mathrm{By}$ varying the random seed used by the Inet graph generator.

${ }^{4}$ False positives are nodes that are identified as stubs by the algorithm, but are not stubs in the ALR. False negatives are stubs in the ALR that are not identifies as stur s by the algorithm.
} 
significant error on the maximum congestion in real graphs employing policy routing. In order to improve the accuracy of labeling edges, we resort to machine learning algorithms.

Employing a good machine learning algorithm for the classification proves to be a tough task, because there is a huge bias towards customer-provider edges in the graphs (roughly $95 \%$ of the edges are customerprovider edges). We use the 3-Nearest Neighbor [14] algorithm for classifying edges as peering or non-peering: each edge in the unlabeled graph is classified as a peering edge if among the three edges most similar to it in the labeled graph, at least two are peering edges. Similarity between edges is judged based on the degrees of their respective end points and neighboring vertices. We measure the accuracy of the procedure by applying it to real graphs and then comparing the classification with true labels.

Our machine learning algorithm gives only $20 \%$ accuracy on peering edges and about $95 \%$ accuracy on customer-provider edges. However, for the purposes of computing the worst congestion in the graph, this low accuracy of labeling is in fact enough. Indeed, as shown in Figure 1(b), labeling real graphs using our algorithm results in an error of less than $10 \%$ in the worst congestion (while employing policy routing) in comparison with the congestion computed on ALRs. More importantly, the growth in congestion is identical in the two cases.

We also report simulation results for congestion in power law trees and exponential topologies. A comparison of the former with power law graphs gives an insight into the significance of density of edges in the graph. The latter model is interesting because most generative models for power law topologies result in exponential distributions in the "fringe" cases. Our tree topologies evolve according to the Preferential Connectivity model [6]. To generate exponential degree distributions, we modify Inet-3.0 to generate an exponential degree distribution first and then add edges in Inet's usual way. For a given $n$, the exponent $\beta$ for the exponential graphs on $n$ nodes is chosen such that the total number of edges in the exponential graph is very close to that of the corresponding power law graph on $n$ nodes $^{5}$. Note that due to a lack of real data for exponential graphs, we do not have a good way of labeling edges and nodes in them. We do not perform experiments with policy routing or the leaf-2-leaf and clout traffic models for them.
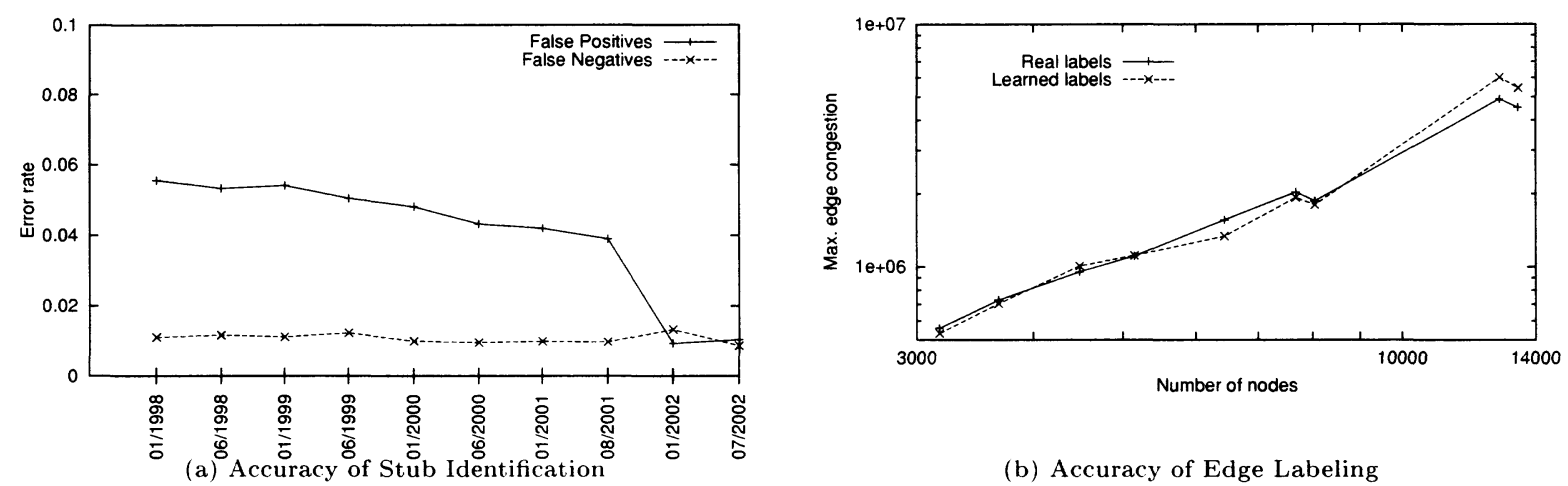

(b) Accuracy of Edge Labeling

Figure 1: Accuracy of heuristics: The graph on the left shows the accuracy of our simple stub identification algorithm. The graph on the right shows the error in the maximum congestion due to our machine-learning based edge-classification algorithm.

\section{Analytical Results}

In this section, we show that the expected maximum edge congestion in a power law graph grows as $\Omega\left(n^{1+\frac{1}{\alpha}}\right)$ with $n$, when we route a unit flow between all pairs of vertices over the shortest path between them.

We consider the Preferential Connectivity Generative Model of Barabasi and Albert [6]. For completeness, we give a brief description of the model. The Preferential Connectivity model is as follows: We use a fixed constant parameter $k$. We start with a complete graph on $k+1$ nodes. We call this set of nodes the core of the graph. Let the graph at time $i$ be denoted $G^{i}$. At time step $i+1$, one node is added to the network.

\footnotetext{
${ }^{5}$ We perform heuristic hill-climbing to estimate the value of the exporent $\beta$ that minimizes error in the number of edges.
} 
This node picks $k$ nodes at random and connects to them. Each vertex $v$ has a probability $\frac{d_{v}^{i}}{D^{i}}$ of getting picked, where $d_{v}^{i}$ is the degree of the vertex at time $i$, and $D^{i}$ is the total degree of all nodes at time $i$.

At the end of $n$ steps, with $k=3$, this process is known to generate a power law degree distribution. We will use the fact that in a power law graph with exponent $\alpha>1$, the maximum degree node has degree $n^{1 / \alpha}$.

In order to show a lower bound on the congestion of a power law graph, our plan is roughly as follows. We consider the edge between the two highest degree nodes in the core- $-s_{1}$ and $s_{2}$. Call this edge $e^{*}$. Then for every vertex $v$ in the graph, we consider the shortest path tree $T_{v}$ rooted at vertex $v$. We show that $\Omega(n)$ such trees contain the edge $e^{*}$. Moreover, in a constant fraction of such trees, there are at least $\Omega\left(n^{\frac{1}{\alpha}}\right)$ nodes in the subtree rooted at edge $e^{*}$.

This gives us the lower bound in the following way: the routes taken by each connection are precisely those defined by the above shortest path trees; thus the congestion on any edge is the sum of congestions on the edge in these shortest path trees. Now the congestion on edge $e^{*}$ in the trees defined above is at least $\Omega\left(n^{\frac{1}{\alpha}}\right)$, and there are $\Omega(n)$ such trees, thus the total congestion on edge $e^{*}$ is at least $\Omega\left(n^{1+\frac{1}{\alpha}}\right)$. Note that $e^{*}$ is not necessarily the most congested edge in the graph, so the maximum congestion could be even worse than $\Omega\left(n^{1+\frac{1}{\alpha}}\right)$. We get the following theorem:

Theorem 1 The expected value of the maximum edge congestion in a power law graph with exponent $\alpha$ grows as $\Omega\left(n^{1+\frac{1}{\alpha}}\right)$ with $n$, when we route a unit flow between all pairs of vertices over the shortest path between them.

We begin with a technical lemma. In the following, the distance between two nodes refers to the number of hops in the shortest path between the two nodes. We will assume that $\alpha>1$.

Lemma 1 Let $r$ be the maximum integer for which at least $\frac{n}{2}$ vertices lie at a distance $r+1$ or beyond from $s_{1}$. Then, $\Omega(n)$ nodes lie within distance $r-1$ of every node in the core of the graph. In particular, for any node in the core, $\Omega(n)$ nodes lie at a distance exactly $r-1$ from it.

Proof: We prove that at least $\Omega(n)$ nodes lie within a distance $r-2$ of $s_{1}$. Then, since all vertices in the core are neighbors of $s_{1}$, these $\Omega(n)$ nodes lie within a distance $r-1$ of any vertex in the core of the graph. We begin by showing that at least $\Omega(n)$ nodes lie within a distance $r$ of $s_{1}$, and then extend this to nodes at distance $r-1$ and $r-2$. Let level $i$ denote the set of nodes at distance exactly $i$ from $s_{1}$.

Remove from the graph all vertices that are at level $r+2$ or higher. The remaining graph has at least $\frac{n}{2}$ vertices, by the definition of $r$. Now, assume that there are at least $\frac{n}{10}$ vertices at level $r+1$, otherwise, we already have $>\frac{2 n}{5}$ nodes in levels 0 through $r$.

Now, let the number of nodes at level $r$ be $x$. All the nodes in level $r+1$ in the residual graph are connected to nodes in level $r$. So, their number is at most the size of the neighbor set of level $r$. Now, in the best possible case, the nodes in level $r$ could be the highest degree nodes in the graph. In this case, the minimum degree of any node in level $r$ is given by $y$ with $n y^{-\alpha}=x$. We get $y=\left(\frac{n}{x}\right)^{\frac{1}{\alpha}}$.

Now, the size of the neighborhood of level $r$ is at most the total degree of nodes in the level. This is given by

$$
\begin{aligned}
\int_{y}^{n^{\frac{1}{\alpha}}} z \alpha n z^{-\alpha-1} d z & =\frac{\alpha n}{\alpha-1}\left(y^{1-\alpha}-n^{\frac{1}{\alpha}-1}\right) \\
& =\frac{\alpha n^{\frac{1}{\alpha}}}{\alpha-1}\left(x^{1-\frac{1}{\alpha}}-1\right)
\end{aligned}
$$

This quantity is at least $\frac{n}{10}$ by our assumption above. Thus we get that $x=\beta n$, where $\beta=$ $\left(\frac{1}{10}\left(1-\frac{1}{\alpha}\right)\right)^{\frac{\alpha}{\alpha-1}}$. This is a constant fraction of $n$.

Now, we can apply the same technique to compute the number of nodes at level $r-1$ and then, $r-2$. We get that the number of nodes at level $r-2$ is at least $\left(\beta^{\alpha}\left(1-\frac{1}{\alpha}\right)\right)^{\frac{\alpha}{(\alpha-1)^{2}}} n$, with $\beta$ as given above.

Let $r$ be the distance defined by the above lemma. Let $V_{r}$ denote the set of nodes that are within distance $r-1$ of every node in the core of the graph. By lemma 1, we have $\left|V_{r}\right|=\Omega(n)$. Now, the proof of the theorem has two parts. The first shows that many trees $T_{v}$ corresponding to $v \in V_{r}$ contain the edge $e^{*}$. 
Lemma 2 The expected number of shortest path trees $T_{v}$, corresponding to leaf nodes $v \in V_{r}$ that contain the edge $e^{*}$ is $\Omega(n)$.

Proof: Consider the tree $T_{v}$ for some node $v \in V_{r}$. This is essentially a breadth first tree starting from $v$. If $s_{1}$ and $s_{2}$ are at the same level in the tree, then the edge $e^{*}$ is not contained in the tree. On the other hand, if the nodes are at different depths in this tree, let $s_{1}$ be closer to $v$ without loss of generality. In this case, one shortest path from $v$ to $s_{2}$ is via $s_{1}$ and since we break ties in favor of paths with high degree nodes, $T_{v}$ will contain this path via $s_{1}$. This implies that $e^{*}$ is contained in the tree. Thus, trees containing $e^{*}$ correspond to those $v$ that are not equidistant from $s_{1}$ and $s_{2}$. We claim that there are $\Omega(n)$ nodes $v \in V_{r}$ that are not equidistant from $s_{1}$ and $s_{2}$. This combined with the fact that a constant fraction of the nodes are leaf nodes implies the result. The latter is immediate from the definition of a power law degree distribution. We now prove the claim.

First, observe that if we pick a random node in the graph, then conditioned on the fact that this node lies at a distance $d-1, d$ or $d+1$ from $s_{2}$, there is at most a constant probability that this node lies at distance $d$ from $s_{2}$. This is because using an argument congruent to that in lemma 1 , we can show that the number of nodes at distance $d-1$ from $s_{2}$ is a constant fraction of the number of nodes at distance $d$.

Now, consider the nodes at distance $r-1$ from $s_{1}$. These are at least $\Omega(n)$ in number (lemma 1 ) and lie in $V_{r}$. Given that a node $v$ is picked from this set, $v$ is at a distance $r-2, r-1$ or $r$ from $s_{2}$. Thus by the above argument, the probability that this node lies at distance $r-1$ from $s_{2}$ is at most a constant. Thus $\Omega(n)$ nodes in this set are not at distance $r-1$ from $s_{2}$ and we are done.

Next we prove that in any tree $T_{v}$ corresponding to a leaf $v \in V_{r}$ containing $e^{*}, e^{*}$ has a high congestion.

Lemma 3 Let $T_{v}$ be a shortest path tree, corresponding to a leaf node $v \in V_{r}$, that contains the edge $e^{*}$. Then the expected congestion on edge $e^{*}$ in this tree is $\Omega\left(n^{1 / \alpha}\right)$.

Proof: Without loss of generality, let $s_{1}$ be closer to $v$ than $s_{2}$. We will show that the degree of $s_{2}$ in $T_{v}$ is $\Omega\left(n^{1 / \alpha}\right)$. This will imply the result.

Let $d$ be the distance between $v$ and $s_{2}$. Note that $d \leq r-1$. First we show that $\Omega(n)$ nodes lie at depth $d+1$ or beyond in the tree. To see this, note that the number of nodes at depth less than $r-1$ is at most the number of nodes within distance $r-1$ from $s_{1} .{ }^{6}$ The remaining nodes, that are at least $\left(\frac{1}{2}-\beta\right) n$ by lemma 1 , where $\beta=\left(\frac{1}{10}\left(1-\frac{1}{\alpha}\right)\right)^{\frac{\alpha}{\alpha-1}}$, lie at depth $d+1$ or beyond.

Now, the neighbors of $s_{2}$ are at hops $d-1, d$ or $d+1$ in the tree $T_{v}$. Consider removing all edges incident on $s_{2}$ except for $e^{*}$. Then, the neighbors of $s_{2}$ that are on level $d-1$ or $d$ of the tree, remain at the same level, whereas those that are at level $d+1$ may move farther from $v$.

Now, let us consider putting the edges incident on $s_{2}$ back into the graph conditioned on the fact that $s_{2}$ should stay at level $d$ in the tree. This means that any node that is at level $d-1$ or beyond can connect to $s_{2}$. Among these nodes, consider those that entered the graph after step $\frac{n}{2}$. By doing so, we are only ignoring a constant fraction of nodes uniformly from the graph.

Among the remaining nodes on levels $d-1$ and beyond, the probability that these nodes formed an edge with $s_{2}$ at the time they entered the graph lies between $n^{\frac{1}{\alpha}-1}$ and $\left(\frac{n}{2}\right)^{\frac{1}{\alpha}-1}<2 n^{\frac{1}{\alpha}-1}$, because the size of the graph varies between $\frac{n}{2}$ and $n$ nodes. Thus, the probability that any such node connects to $s_{2}$ is within a factor of less than 2 of the probability for any other node. Now as mentioned above, the number of nodes at level $d+1$ and beyond is at least some constant, say $\gamma$, times the number of nodes in levels $d-1$ and $d$. Consequently, the expected number of nodes in levels $d+1$ and beyond that connect to $s_{2}$ is at least a constant fraction (around $\frac{\gamma}{2}$ ) of the expected number of nodes in levels $d-1$ and $d$ that connect to $s_{2}$. This is a constant fraction of the total degree of $s_{2}$, which is $\Omega\left(n^{\frac{1}{\alpha}}\right)$. Thus we get the result.

\subsection{Experimental Support}

In this section, we report results from experiments conducted to validate that the theoretical results obtained above hold not just for the Preferential Connectivity Model, but also for Internet-like graphs generated by Inet-3.0.

\footnotetext{
${ }^{6}$ This is easily seen to be the case by the definition of $r$ and lemma 1 when $r \leq 4$, because $v$ is a leaf. Moreover, our simulations show that $r \leq 4$ for all values of $n$.
} 


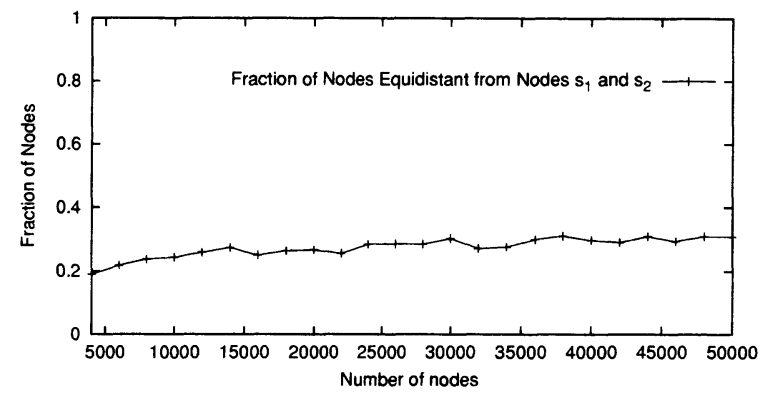

Figure 2: Fraction of shortest path trees that do not contain the edge $e^{*}$.

Unfortunately, the graphs generated by Inet-3.0, have different values of $\alpha$ for different $n$. This is consistent with the observed behavior of the Internet, that $\alpha$ decreases with time. (We discuss this in further detail in the following section). In order to validate our theoretical results and observe the asymptotic behavior of congestion for a fixed value of $\alpha$, we modify the Inet-3.0 code, for the purposes of this section, so that it always uses a fixed value of $\alpha=1.23$, instead of recalculating it for every value of $n$. Each reported value is an average over multiple runs of the simulation, corresponding to different random seeds used for generating the graphs.

Figure 2 plots the fraction of nodes that are equidistant from $s_{1}$ and $s_{2}$. Note that this fraction always lies below 0.4 and is consistent with our result in lemma 2 that at least a constant fraction of the trees, $\frac{n}{2}$ in this case, contain the edge $e^{*}$.

Figure 3 compares the degrees of the two highest degree nodes in the graph to their corresponding degrees in the shortest path tree corresponding to some random node $v$. We find that the ratio of the two degrees for $s_{1}$ is consistently above 0.9 . Similarly, the ratio of the two degrees for $s_{2}$ is always above 0.8 and increasing. This is consistent with the findings of lemma 3.

Finally, we plot the maximum congestion in graphs generated by Inet-3.0, as a function of the number of nodes in the graph, in Figure 4. Note that the maximum congestion scales roughly as $n^{1.8}$, which is exactly $n^{1+1 / \alpha}$ for the given value of $\alpha$. This corroborates our finding in Theorem 1 .

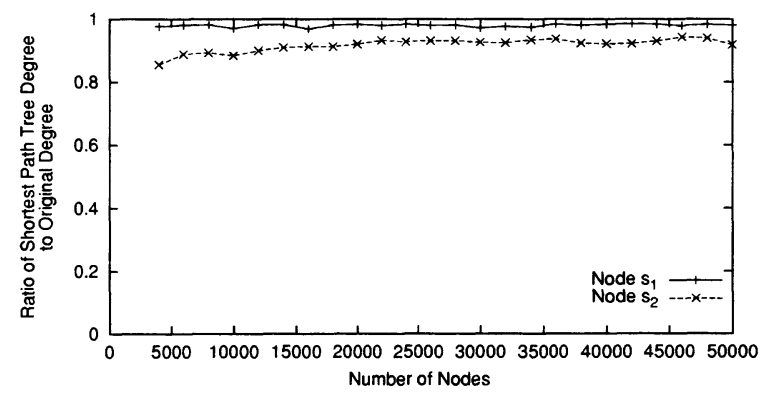

Figure 3: Congestion on edge $e^{*}$ in a random shortest path tree: This figure plots the ratio of degrees of $s_{1}$ and $s_{2}$ in the graph to their degrees in a random shortest path tree.

\section{Simulation Results}

In this section, we present the results from our simulation study over Inet-generated graphs. Henceforth, we shall use the graphs generated by Inet 3.0 as is, that is, we do not alter the way Inet chooses $\alpha$ to depend on $n$. (Recall that, in contrast, the simulation results in the previous section use the modified Inet 3.0 code which employs the same value of $\alpha$ for all $n$. We do not show results for such graphs.) In what follows, we 


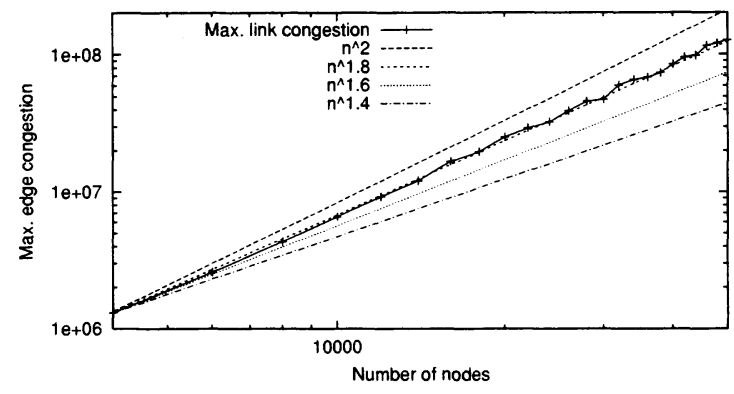

Figure 4: Maximum congestion as a function of $n$, in Inet-3.0 generated graphs, with $\alpha=1.23$. The figure also plots four other functions to aid comparison $-n^{1.4}, n^{1.6}, n^{1.8}, n^{2}$.

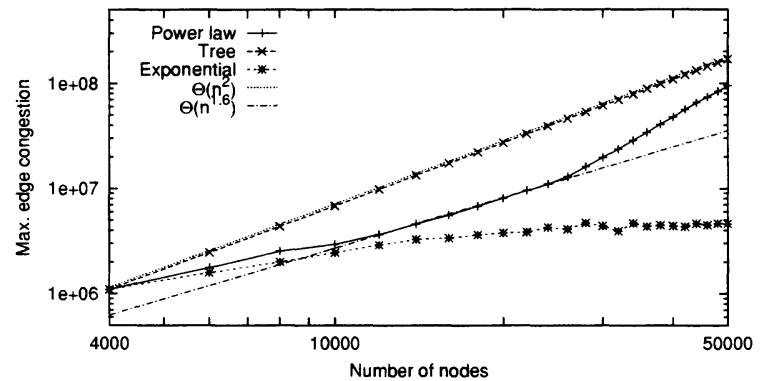

(a) Maximum edge congestion

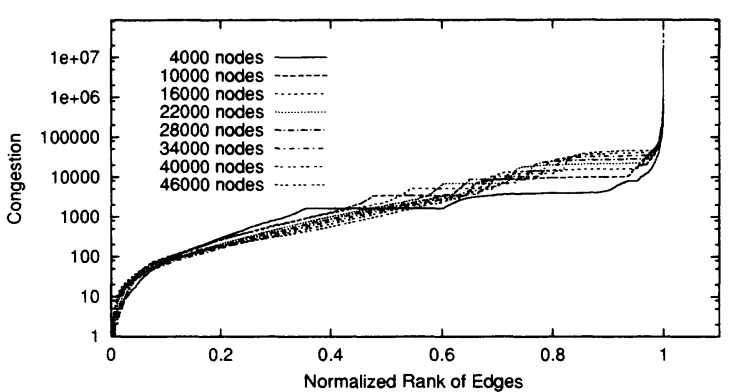

(b) Distribution of edge congestion

Figure 5: Edge congestion with shortest path routing and any-2-any communication: The figure on the left shows the maximum edge congestion. The figure on the right shows the distribution of congestion over all links, with the number of links normalized to 1 in each case. The figure on the left also plots the worst congestion for exponential graphs and preferential connectivity trees.

first show results for shortest-path routing, followed by policy-based routing. In both cases, we first present results for the any-2-any communication model, then for the leaf-2-leaf model and finally for the clout model.

\subsection{Shortest-Path Routing}

Figure 5(a) shows the maximum congestion in power law graphs generated by Inet-3.0 as a function of the number of nodes. We use the any-2-any model of communication here. From the trend in the graph, it is clear that the maximum congestion in Internet-like graphs scales worse that $n^{1+\Omega(1)}$. Notice also that the slope of the maximum congestion curve is slightly increasing. This may be explained as follows. As mentioned earlier, Inet-3.0 chooses the exponent of the power law degree distribution as a function of the number of nodes $n: \alpha=a t+b$, where $t=\frac{1}{s} \log \frac{n}{n_{0}}, a=-0.00324, b=1.223, s=0.0281$ and $n_{0}=3037 .{ }^{7}$ Notice that the absolute value of $\alpha$ decreases as $n$ increases, and so, as our lower bound of $\Omega\left(n^{1+1 / \alpha}\right)$ suggests, the slope of the function on a $\log -\log$ plot should steadily increase. In fact around $n=28000, \alpha$ becomes less than 1 and at this point we expect the curve to scale roughly as $n^{2}$, which is the worst possible rate of growth of congestion.

The figure also shows the maximum congestion in power law trees and exponential graphs. The power law trees we generate, have the exponent $\alpha$ between 1.66 and 1.8 , the value increasing with the number of nodes in the tree. These exponents are significantly higher than those of the corresponding power law graphs. Notice that the edge congestion on power law trees grows much faster as compared to graphs which is expected since trees have much fewer edges. Our lower bound on the maximum congestion, which holds

\footnotetext{
${ }^{7} a, b$ and $s$ are empirically determined constants. $n_{0}$ is the number of ASes in the Internet in November 1997.
} 
equally well for trees satisfying power law degree distributions, predicts the slope of the curve for trees to be at least 1.5 , which is consistent with the above graph.

On the other hand, we notice that edge congestion in exponential graphs is much smaller compared to power law graphs. In fact, edge congestion in exponential graphs has a less than linear growth (i.e., scales as $O(n)$ ). This could be explained intuitively as follows: Recall that for each $n$, we choose the exponent $\beta$ of the exponential distribution so as to match the total number of edges of the corresponding $n$-node power law graph. Because the power law distribution has a heavier tail compared to the exponential distribution, the latter has more edges incident on low degree nodes. Consequently, low degree vertices in an exponential graph are better connected to other low degree vertices. Edges incident on low degree nodes "absorb" a large amount of congestion leading to lower congestion on edges incident on high degree nodes. As $n$ increases the degree distribution becomes more and more even, resulting in a very slow increase in congestion.

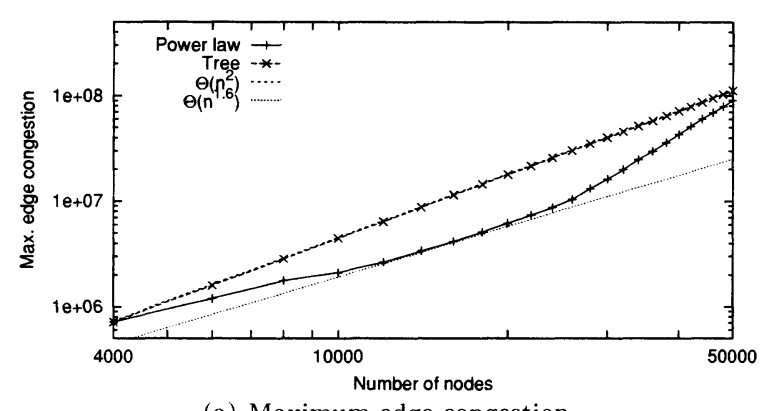

(a) Maximum edge congestion

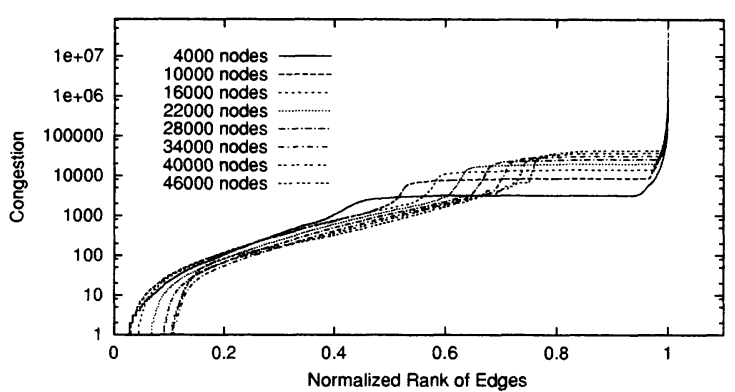

(b) Distribution of edge congestion

\section{Figure 6: Edge congestion with shortest path routing and leaf-2-leaf communication}

In Figure 5(b), we show the congestion across all links in a power law graph for varying numbers of nodes. Notice that at higher numbers of nodes, the distribution of congestion becomes more and more uneven.

The corresponding set of graphs for the leaf-2-leaf communication model is shown in Figure 6. The worst congestion is consistently about 0.8 times the worst congestion for the any-2-any model (not explicitly shown in the graph). The congestion across all the edges, plotted in Figure 6(b), also displays a similar trend as for the any-2-any model - the distribution becomes more uneven as the number of nodes increases.

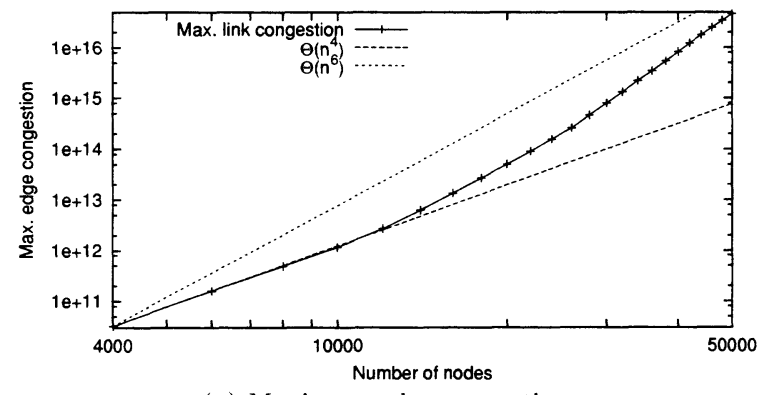

(a) Maximum edge congestion

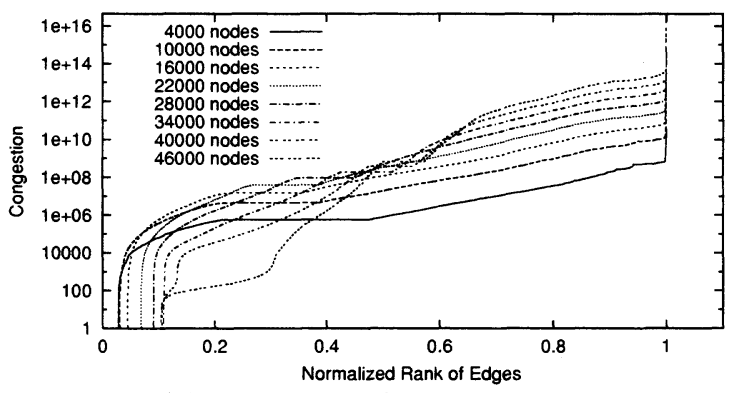

(b) Distribution of edge congestion

Figure 7: Edge congestion with shortest path routing and clout model of communication

The results for the clout model are more interesting with the resulting maximum congestion in the graph scaling much worse than before. Indeed, as Figure 7(a) shows, the maximum congestion scales worse than $n^{5}$. This is because the total traffic in the graph also grows roughly as $O\left(n^{4}\right)$. Again, as with the any-2-any model, the smaller absolute values of $\alpha$ in the graphs generated by Inet-3.0 for larger values of $n$ is a plausible explanation for the increasing slope of the curve.

The graph of the congestion across all edges in this model, shown in Figure 7(b), is equally interesting. Compared to Figure 6(b) of the leaf-2-leaf model, Figure 7(b) looks very different. Visual inspection of 
the two figures reveals that the unevenness in congestion is much more pronounced in the clout model of communication. To summarize, the non-uniform traffic demand distribution in the Internet only seems to exacerbate the already poor congestion scaling of the Internet.

\subsubsection{Shortest Path Routing Variations}

As mentioned in Section 3.1, we chose shortest path routing with three possible variations: favoring paths on which the nodes have higher degrees, favoring paths where the nodes have lower degrees, and choosing a random shortest path when there is a choice of more than one.

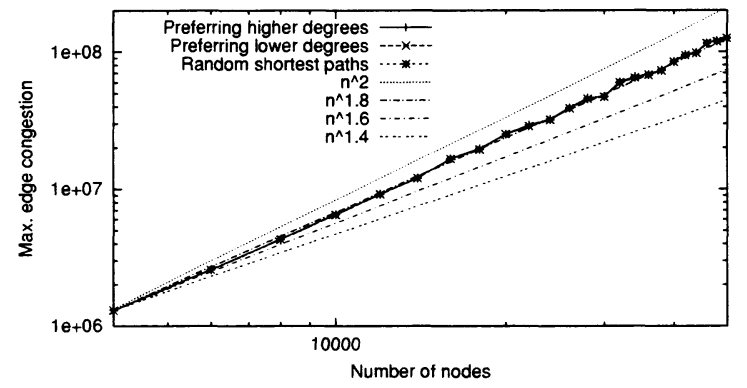

Figure 8: Edge congestion with shortest path routing and any-2-any communication, with $\alpha=$ 1.23. The figure plots the three different variations of breaking ties in shortest path routing.

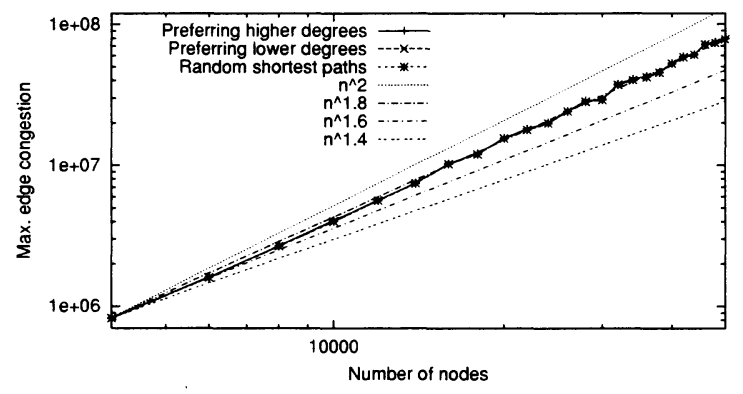

Figure 9: Edge congestion with shortest path routing and leaf-2-leaf communication, with $\alpha=$ 1.23. The figure plots the three different variations of breaking ties in shortest path routing.

In assessing the deviation between the three different means of tie-breaking for shortest path routing, we chose to simulate these graphs by setting $\alpha$ to be a constant value in Inet 3.0 and compared the resulting relations between maximum edge congestion and the number of nodes.

As Figure 8 depicts, the difference between the three types of tie-breaking methods is hardly noticeable. In fact the graphs seem to coincide, but upon close inspection of the generated data slight differences are noted due to variation. The same case holds true for Figures 9 and 10. We thus conclude that our scheme of breaking ties by favoring paths containing nodes with greater degree does not skew our results.

\subsection{Policy-Based Routing}

Figure 11 shows the maximum edge congestion for the three communication models when policy based routing is used. For the any-2-any and leaf-2-leaf models, shown in Figure 11(a), the maximum edge congestion scales almost identically to that for shortest path routing (compared with Figure 5(a) and 6(a)). However, somewhat surprisingly, for the clout model, congestion under policy based routing scales only as $n^{3}$ compared to over $n^{5}$ for shortest-path routing. 


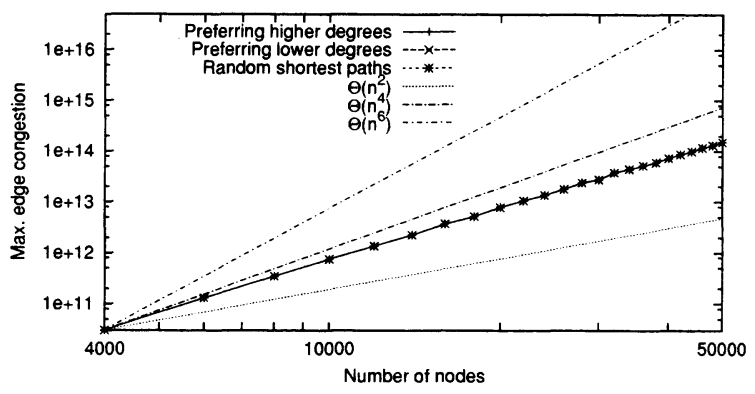

Figure 10: Edge congestion with shortest path routing and clout model of communication, with $\alpha=1.23$. The figure plots the three different variations of breaking ties in shortest path routing.

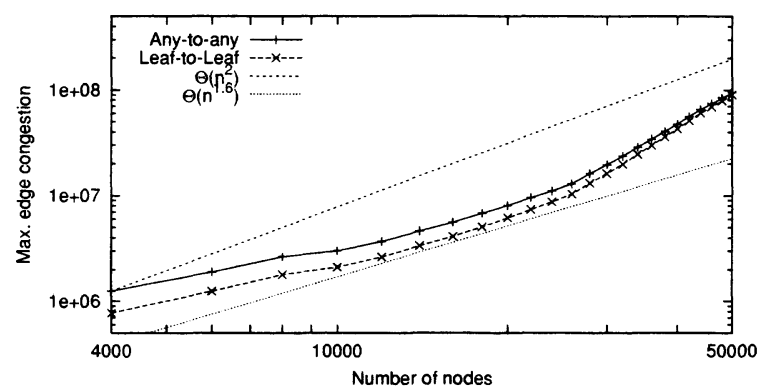

(a) Any-2-any and Leaf-2-leaf communication

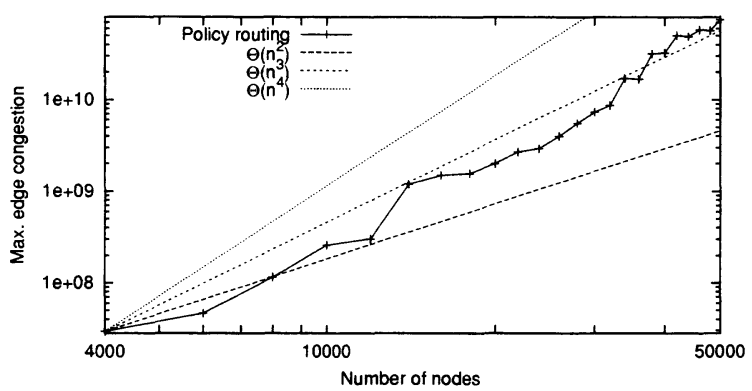

(b) Clout model

Figure 11: Maximum Edge congestion with policy-based routing in HLSs

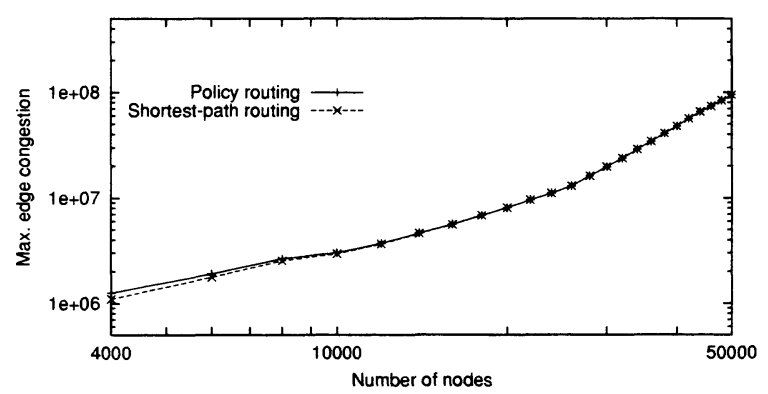

(a) Edge congestion on synthetic graphs

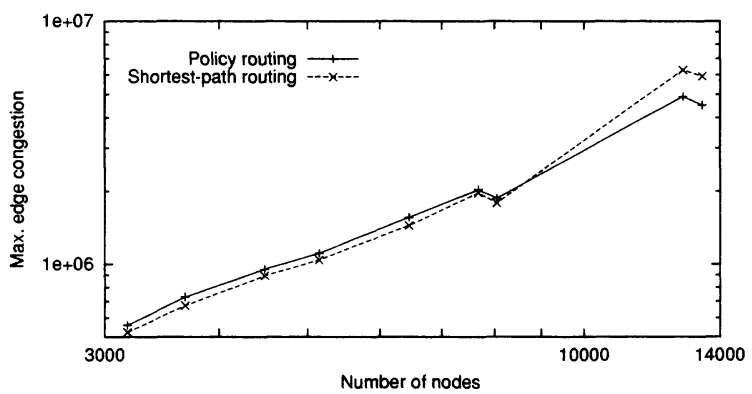

(b) Edge congestion on real graphs

Figure 12: Comparison of edge congestion for shortest path and policy based routing in the any-2-any model

Figure 12(a) compares maximum congestion obtained for policy routing to that for shortest path routing. Notice that the two curves are almost overlapping, although policy routing seems to be slightly worse when the graph is small and gets better as the graph grows larger. This observation can be explained as follows: policy routing disallows certain paths from being used and could thus, in general, force connections to be routed over longer paths. This would increase the overall traffic in the network leading to higher congestion, especially for smaller numbers of nodes. However, as the size of the graph grows, there are more and more shortest paths available. As a result, the constraints placed by policy-based routing might not have any significant impact on the path lengths in the graph. In fact, at higher numbers of nodes, policy routing could provide better congestion properties, albeit only marginally different, than shortest path routing. This is because while shortest path routing always picks paths that go over high degree nodes, a fraction of these 
paths might not be allowed by policy routing as they could involve more than one peering edge. In this case, policy routing moves traffic away from the hot-spots, thereby, partially alleviating the problem.

In order to verify that the above observation is not just an artifact of our machine learning-based labeling algorithms, we plot the same curves for ALRs in Figure 12(b). These display exactly the same trend-policy routing starts out being worse than shortest path, but gets marginally better as $n$ increases. To summarize, policy routing does not worsen the congestion in Internet like graphs, contrary to what common intuition might suggest. In fact, policy routing might perform marginally better than shortest path routing.

\section{Discussion}

Our analytical and simulation results have shown that the power law nature of the Internet graph causes the maximum congestion in the network to scale rather poorly $-\Omega\left(n^{1+\Omega(1)}\right)$. As mentioned in Section 1 , this implies that as the Internet grows in its size, the uniform scaling in the capacities of all links in the Internet graph according to Moore's Law, might not be enough to sustain the increasing congestion in the graph. Our results show that the high degree nodes, which are typically in the core of the Internet, will get congested more quickly over time than the edges. In such a situation, to enhance the scaling properties of the network, it might become necessary to either change the routing algorithm employed by the nodes or alter the macroscopic structure of the graph. We address the latter issue in this section.

\subsection{Adding Parallel Network Links}

In this section, we examine ways in which additional links can be placed in the network, so as to contain the effect of bad scaling of the maximum congestion. Specifically, we consider the model in which each link can be replaced by multiple links (between the same pair of nodes) that can share the traffic load ${ }^{8}$. Ideally, we would like to provide sufficient parallel links between a pair of nodes, so that the total congestion on the corresponding edge divided equally among these parallel links, even in the worst case, grows at about the same rate as the size of the network. The number of parallel links between a pair of nodes may need to change as the network grows to achieve this goal. Notice that this change does alter the degree-structure of the graph, but the alteration is only due to increased connectivity between already adjacent nodes ${ }^{9}$. This does not require new edges between nodes that were not adjacent before.

In some ways, the network already incorporates this concept of parallel links. For example, the power law structure of the AS graph only considers the adjacency of ASes: the link between Sprint and AT\&T, for instance, is modelled by a single edge. However, in the real world the Sprint and AT\&T ASes are connected to each other in a large number of places around the world. However, not much is known about the degree of such connectivity in the Internet today.

In order to guide the addition of parallel edges between adjacent nodes, we first observe that there is clear correlation between the average degree and edge congestion. Figure 13 plots the congestion of each edge against the average degree of the nodes on which it is incident, for shortest path routing on an Inet generated graph of 30000 nodes. The form of communication used here is any-2-any. The figure shows that edges incident on high degree nodes have much higher congestion than those incident on lower degree nodes. This suggests that a good choice for the number of parallel links substituting any edge in the graph, could depend on the degrees of nodes which an edge connects.

We examine several ways of adding parallel links based on the above observation. In particular, we let the number of links between two nodes be some function of the degrees of the two nodes and we consider the following functions: (1) sum of degrees of the two nodes, (2) product of the degrees of the two nodes, (3) maximum of the two degrees and, (4) minimum of the two degrees. For each of these functions, we compute the maximum relative congestion, that is, the maximum over all edges, of the congestion on the edge divided by the number of parallel links corresponding to each edge. In what follows, we show simulation results about how the maximum relative congestion scales for shortest path routing on power law graphs within the any-2-any model of communication.

\footnotetext{
${ }^{8}$ For results on alternate methods of alleviating congestion, please refer to a full version of this paper [?].

${ }^{9}$ Note that the routing is still done based on the original degrees of nodes.
} 


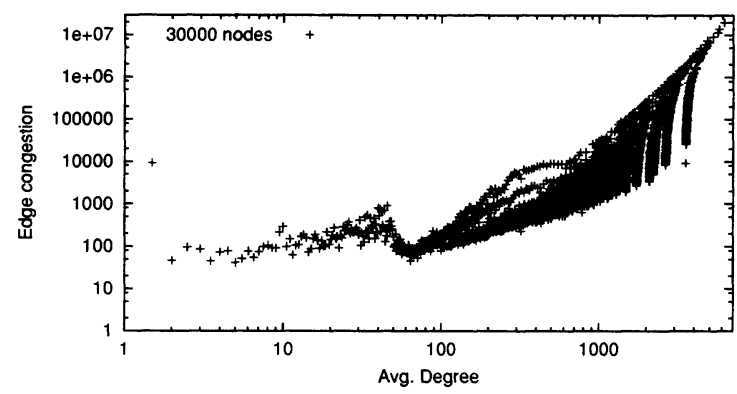

Figure 13: Edge Congestion versus the average degree of the nodes incident on the edge (any2-any model with shortest path routing). The congestion is higher on edges with a high average degree.

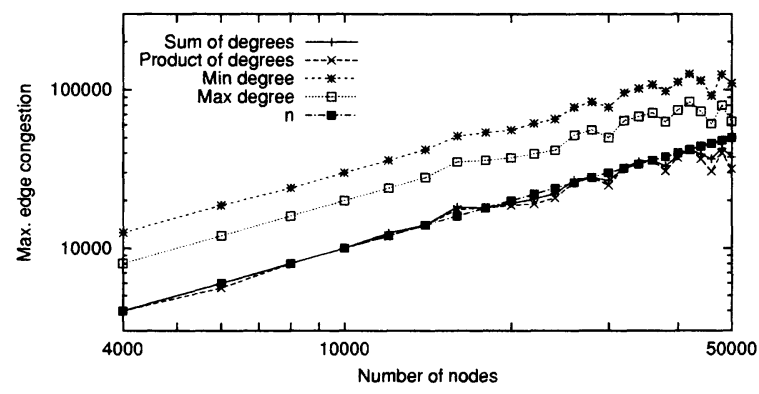

Figure 14: Maximum relative congestion for shortest path routing, any-2-any model, when parallel links are added to the graph using the sum, product, max and min functions.

The results are shown in Figure 14. Notice that, surprisingly, when parallel links are added according to any of the above four functions the maximum relative congestion in the graph scales linearly. This implies that adding parallelism in the edges of Internet-like graphs according to the above simple functions is enough to ensure that the scaling of link capacities in the Internet according to Moore's law can maintain uniform levels of congestion in the network and avoid any potential hot-spots.

\subsection{Random Edge Addition}

Next we consider altering the structure of the graph, by adding random edges to the topology. Although we do not present results for these, we discuss the implications of random edge addition. For instance, a percentage of node pairs that are not connected in the current topology would have an edge added between them. This could potentially alleviate congestion due to the fact that some of the stub nodes would not need to route their traffic through the core of the graph to communicate to other stub nodes.

Another model that is being considered is one where edges are added between nodes with high degree. Consider the core of the topology, this is where the highest degree nodes tend to be. We would then like to examine randomly adding edges between two nodes of high degree that are not connected in the original topology. This could help reroute some of the traffic that goes through the core, particularly through the nodes with very high degreę. If edges were added between the high-degree nodes, perhaps the traffic could become more distributed leading to a reduction in the maximum edge congestion and better scaling of the worst edge congestion in the topology. 


\section{Summary}

In this paper, we addressed the question of how the worst congestion in Internet-like graphs scales with the graph size. Using a combination of analytical arguments and simulations studies, we show that the maximum congestion scales poorly in Internet-like power law graphs. Our simulation results show that the non-uniform demand distribution between nodes only exacerbates the congestion scaling. However, we find, surprisingly, that policy routing may not worsen the congestion scaling on power law graphs and might, in fact, be marginally better when compared to shortest-path routing.

Our results show that, with the current trend of the growth of the Internet, some locations in the network might eventually become perpetual hot-spots. Fortunately, however, there is an intuitively simple fix to this problem. Adding parallel links between adjacent nodes in the graph according to simple functions of their degrees will help the maximum congestion in the graph scale linearly. In this case, it might not be necessary for some links in the graph to grow in capacity at a faster rate than the others.

\section{References}

[1] BGP Tables from the University of Oregon RouteViews Project. http://moat.nlanr.net/AS/data.

[2] National laboratory for applied network research. Routing data. http://moat.nlanr.net/Routing/rawdata/.

[3] Aiello, W., Chung, F., ANd Lu, L. Random evolution in massive graphs. In FOCS (2001), pp. 510519.

[4] Albert, R., and Barabasi, A.-L. Topology of evolving networks: local events and universality. Physical Review Letters 85(24) (2000), 5234-5237.

[5] Aumann, Y., And Rabani, Y. An $o(\log k)$ approximate min-cut max-flow theorem and approximation algorithm. SIAM Journal on Computing 27(1) (1998), 291-301.

[6] Barabasi, A.-L., And Albert, R. Emergence of scaling in random networks. Science 286 (1999), 509-512.

[7] Fabrikant, A., Koutsoupias, E., and Papadimitriou, C. Heuristically optimized trade-offs: A new paradigm for power laws in the Internet. In ICALP (2002), pp. 110-122.

[8] Faloutsos, M., Faloutsos, P., and Faloutsos, C. On power-law relationships of the Internet topology. In Proceedings of the SIGCOMM '9g Symposium on Communications Architectures and Protocols (1999), pp. 251-262.

[9] GAO, L. On inferring autonomous system relationships in the Internet. IEEE/ACM Transactions on Networking 9(6) (December 2001).

[10] Gkantsidis, C., Saberi, A., And Mihail, M. Conductance and congestion in power law graphs. In Proceedings of the ACM Sigmetrics 2003 (2003).

[11] Leighton, F. T. Introduction to Parallel Algorithms and Architectures: Arrays, Trees, Hypercubes. Morgan Kaufmann Publishers, 1992.

[12] LeOnardi, S. On-line network routing. In Online Algorithms - The State of the Art (1998), Springer, pp. 242-267.

[13] Medina, A., Lakhina, A., Matta, I., and Byers, J. Brite: An approach to universal topology generation. In MASCOTS (2001).

[14] Mitchell, T. M. Machine Learning. McGraw-Hill Companies, Inc., 1997. 

[15] Okamura, H., And Seymour, P. Multicommodity flows in planar graphs. Journal of Combinatorial Theory B 31 (1981), 75-81.

[16] Park, K., AND LeE, H. On the effectiveness of route-based packet filtering for distributed DoS attack prevention in power-law Internets. In Proceedings of the SIGCOMM '01 Symposium on Communications Architectures and Protocols (San Diego, California, August 2001).

[17] Subramanian, L., Agarwal, S., Rexford, J., and Katz, R. H. Characterizing the Internet hierarchy from multiple vantage points. In Proceedings of IEEE INFOCOM (June 2002).

[18] Tangmunarunkit, H., Govindan, R., Jamin, S., Shenker, S., and Willinger, W. Network topology generators: Degree-based vs structural. In Proceedings of the SIGCOMM '02 Symposium on Communications Architectures and Protocols (Pittsburgh, Pennsylvania, August 2002).

[19] Winick, J., AND JAMIN, S. Inet-3.0: Internet topology generator. Tech. rep., University of Michigan, 2002. Technical Report, CSE-TR-456-02. 
